\title{
Evidence that fibroblasts derive from epithelium during tissue fibrosis
}

\author{
Masayuki Iwano, ${ }^{1}$ David Plieth, ${ }^{1}$ Theodore M. Danoff, ${ }^{2,3}$ Chengsen Xue, ${ }^{1}$ \\ Hirokazu Okada, ${ }^{3,4}$ and Eric G. Neilson ${ }^{1,3}$ \\ ${ }^{1}$ Department of Medicine, and Department of Cell and Developmental Biology, Vanderbilt University, \\ Nashville, Tennessee, USA \\ ${ }^{2}$ GlaxoSmithKline, Philadelphia, Pennsylvania, USA \\ ${ }^{3}$ Department of Medicine, University of Pennsylvania, Philadelphia, Pennsylvania, USA \\ ${ }^{4}$ Department of Nephrology, Saitama Medical College, Irumagun, Japan
}

\begin{abstract}
Interstitial fibroblasts are principal effector cells of organ fibrosis in kidneys, lungs, and liver. While some view fibroblasts in adult tissues as nothing more than primitive mesenchymal cells surviving embryologic development, they differ from mesenchymal cells in their unique expression of fibroblast-specific protein-1 (FSP1). This difference raises questions about their origin. Using bone marrow chimeras and transgenic reporter mice, we show here that interstitial kidney fibroblasts derive from two sources. A small number of $\mathrm{FSP}^{+}, \mathrm{CD}^{4} 4^{-}$fibroblasts migrate to normal interstitial spaces from bone marrow. More surprisingly, however, $\mathrm{FSP}^{+}$fibroblasts also arise in large numbers by local epithelial-mesenchymal transition (EMT) during renal fibrogenesis. Both populations of fibroblasts express collagen type I and expand by cell division during tissue fibrosis. Our findings suggest that a substantial number of organ fibroblasts appear through a novel reversal in the direction of epithelial cell fate. As a general mechanism, this change in fate highlights the potential plasticity of differentiated cells in adult tissues under pathologic conditions.
\end{abstract}

J. Clin. Invest. 110:341-350 (2002). doi:10.1172/JCI200215518.

\section{Introduction}

Cell fate pathways for epithelial tissues have overlapping complexities on many levels (1). Pathway integration ultimately determines the migration and interaction of progenitor cells under the control of genetic and morphogenic cues, the timed partitioning of cellular determinants, and plasticity among lineages until terminal differentiation shapes final structure and function $(2,3)$. With evolving tissue maturity, epithelial units organize as repeating structures, and fibroblasts come to reside in the interstitial spaces that form between functional units. Unfortunately, the order and assembly of these patterned events are not well understood $(4,5)$; for that matter, not all cells have been studied. The origin of interstitial fibroblasts, for example, has been largely overlooked, and their lineage is inconclusive (6). We undertook the present study because recent availabil-

Received for publication March 25, 2002, and accepted in revised form June 11, 2002.

Address correspondence to: Eric G. Neilson, Department of Medicine, D-3100 Medical Center North, Vanderbilt University Medical Center, Nashville, Tennessee 37232-2358, USA

Phone: (615) 322-3146; Fax: (615) 343-9391;

E-mail: Eric.Neilson@Vanderbilt.edu.

Masayuki Iwano and David Plieth contributed equally to this work.

Conflict of interest: No conflict of interest has been declared. Nonstandard abbreviations used: marrow stromal cells (MSCs); epithelial-mesenchymal transition (EMT); fibroblast-specific protein-1 (FSP1); green fluorescent protein (GFP); unilateral ureteral obstruction (UUO); bone marrow lining cell (BMLC). ity of new fibroblast markers has reduced the difficulty in addressing this issue $(7,8)$.

Two hypotheses emerge regarding the origin of adult fibroblasts. One hypothesis argues that marrow stromal cells (MSCs) are progenitors for tissue fibroblasts that then shuttle through the circulation to populate peripheral organs $(6,9-11)$. While MSCs can migrate to remote tissues and clearly develop a fibroblastic phenotype in culture (6), no evidence exists to show they engage in tissue fibrosis after migration. In fact, most of the recent interest in MSCs focuses on their capacity to give rise to more differentiated cells in nonhematogenous organs $(5,12,13)$. A second hypothesis favors epithelial-mesenchymal transition (EMT) in the local formation of interstitial fibroblasts from organ epithelium (7). While many neoteric cell lineages migrate during embryogenesis to new locations using a fate pathway that involves EMT $(14,15)$, such transitions in mature tissues are less well appreciated. However, transitions do occur among adult cells (5), particularly during oncogenesis (16) and fibrotic tissue repair following injury - a process known as fibrogenesis $(7,17)$.

The appeal of an argument for EMT in the formation of fibroblasts is its simplicity; fibroblast dispersal in local interstitial spaces is assured by local epithelium, particularly when fibroblasts are needed for fibrogenesis. Indirect support for this notion stems from earlier work that identified fibroblast-specific protein-1 (FSP1) as an EMT marker in cultured epithelial cells undergoing transition to fibroblasts (18), as well as histologic evidence in vivo that epithelial units expressing FSP1 
disaggregate as organ tissues dedifferentiate during the early stages of fibrogenesis $(7,19)$.

Epithelial cells sit on and attach to basement membranes that provide context and architectural stability for the cell-cell contact emblematic of this phenotype. When basement membrane is damaged by proteases or disrupted by alterations in assembly, epithelia begin to express cytokines that initiate EMT (20). Growth factors such as TGF- $\beta$, EGF, and FGF- 2 facilitate EMT by binding epithelial receptors with ligand-inducible intrinsic kinase activity $(16,21,22)$. The activation of Ras and Src pathways (16) and a shift in the balance of small GTPase activity (23) provide important transcriptional signals for loss of adhesion (24) and induction of EMT in cultured cells. In performing these functions, TGF- $\beta$ and EGF also induce the expression of FSP1 in transitioning tubular epithelium (18). FSP1 is a fibroblast-specific protein in the $\mathrm{S} 100$ class of cytoplasmic, calcium-binding proteins (7). The members of this family have been implicated in microtubule dynamics, cytoskeletal membrane interactions, calcium signal transduction, p53-mediated cell cycle regulation, and cellular growth and differentiation. While the precise function of FSP1 and its homologues is not entirely clear, its interaction with non-muscle myosin II, tropomyosin, actin, or tubulin, and the inducibility of rearrangements in F-actin stress fibers suggest that FSP1 may be associated with mesenchymal cell shape and motility. Inhibition of EMT in cultured cells by blocking FSP1 expression implies a direct role in reshaping cytoskeletal architecture (18).

While EMT is well described in cultured cells, it remains to be shown whether such events occur in adult vertebrate tissues. Furthermore, although fibroblasts readily proliferate in culture when bombarded with cytokines and serum, they are generally quiescent in normal tissue. In preliminary in vivo experiments in normal mice expressing a transgene in tissue fibroblasts that encodes for thymidine kinase under the control of the FSP1 promoter (25), FSP1+ fibroblasts were not reduced in number by DNA chain termination until they randomly entered the cell cycle after more than 4-6 weeks of exposure to nucleoside analogues (data not shown). Therefore, in order to examine their in vivo derivation in a shorter time frame, we studied the appearance of new fibroblasts in a model of experimental renal fibrosis using specific protein markers. This approach, described below, also allowed us to evaluate the functional contribution of new fibroblasts identified from multiple sources.

\section{Methods}

Transgenic mice. Two sets of transgenic mice were constructed for these experiments. In the $\gamma$ GT.Cre mouse, the Cre transgene was removed from pMCCre (26) by digestion with MluI, and the overhang ends were blunted with Klenow fragment. A second vector, prGT$\mathrm{GH}$, containing the rat $\gamma G T$ promoter and a human growth hormone polyadenylation site (27), was digest- ed at the XbaI site between the promoter and polyadenylation sequences, and the ends were also blunted with Klenow fragment. The blunted Cre transgene was then ligated to the blunted $\gamma G T$ promoter plasmid, and positive clones were selected for proper orientation. The chosen construct was then linearized using NotI and HindIII, and purified DNA was injected into $\mathrm{B} 6 \times \mathrm{SJL}$ zygotes. The resulting progeny were crossed to Balb/c mice and selected by Southern blot and $\operatorname{PCR}(7,8)$. The second transgene inserted into the FSP1.GFP mouse was assembled using pEGFP-N1 (Clontech, Palo Alto, California, USA) as a convenience vector. After deletion of the CMV promoter by digestion with AgeI and PstI and inserting a linker with XhoI and SfiI restriction sites, the vector was digested with EcoRI $\left(5^{\prime}\right)$ and BamHI $\left(3^{\prime}\right)$ in the region $5^{\prime}$ of the green fluorescent protein (GFP) gene. The EcoRI-to-NcoI FSP1 promoter fragment, with the NcoI site blunted by mung bean nuclease to remove the first ATG, was excised from a donor vector, FSP1.TK, $(7,8)$ using EcoRI ( $\left.5^{\prime}\right)$ and BamHI $\left(3^{\prime}\right)$ and then inserted into the GFP vector. Digestion of the final product with EcoRI and DraIII, (a site downstream of the polyadenylation signal), produced a linearized product for injection into B6D2 zygotes. Potential recombinant progeny were crossed to a Balb/c background and selected by Southern blot and PCR analysis of tail DNA. After secondgeneration mating, expression of GFP via the transgenic promoter is visible in the eyes of the mice under Woods illumination (see Figure $3 \mathrm{~b}$ ). Transgenic protocols were approved by the Instructional Animal Care and Use Committee (IACUC) at the University of Pennsylvania and Vanderbilt University.

DNA identification. DNA was extracted from normal kidneys and liver harvested from $\gamma$ GT.Cre, R26R, and $\mathrm{R} 26 \mathrm{R} \times \gamma \mathrm{GT}$.Cre mice $(8,28)$. PCR was performed in 50 $\mu \mathrm{l}$ of solution containing DNA, standard reaction buffer, dNTPs (0.2 mM), 2.5 U Taq DNA polymerase (Roche Molecular Biochemicals, Indianapolis, Indiana, USA), and $1.0 \mathrm{mM}$ of each of two primers. The following PCR primers and annealing temperatures were used. R26R: Rosa 5'(AAAGTCGCTCTGAGTTGTTAT), Rosa $3^{\prime}$ (GCGAAGAGTTTGTCCTCAACC) $\quad 62^{\circ} \mathrm{C} ; \quad \gamma$ GT.Cre: Cre5'(AgGtgTAGagaAGgCACTTAGC), Cre3'(CTAATCGCCATCTTCCAGCAGG), $63^{\circ} \mathrm{C}$; unfloxed: Rosa $5^{\prime}$ (above), LacZ $3^{\prime}$ (TGTGCTGCAAGGCGATTAAGTTGG), 58 ${ }^{\circ} \mathrm{C}$; FSP1.GFP: FSP1 5' (GTGATTTGGGTCATGCTCAG), GFP $3^{\prime}$ (GAACAGCTCCTCGCCCTTGC), $58^{\circ} \mathrm{C}$; control: FSP1 $5^{\prime}$ (above), FSP1 $3^{\prime}$ (CATTGCACATCATGGCAATG), $58^{\circ} \mathrm{C}$. Cycle programs were performed in either a Hybaid Omni (Hybaid, Middlesex, United Kingdom) or a Perkin-Elmer (Perkins-Elmer, San Jose, California, USA) thermocycler. All primers were started with 3 minutes at $95^{\circ} \mathrm{C}$, followed by 30 cycles of 1 minute at $95^{\circ} \mathrm{C}$, 1 minute at the primer's appropriate annealing temperature, and 1 minute at $72^{\circ} \mathrm{C}$, finishing with $7 \mathrm{~min}$ utes at $72^{\circ} \mathrm{C}$. Products were analyzed by electrophoresis in $1.5 \%$ agarose using TAE buffer and a 100-bp ladder sizing standard. 
RT-PCR amplifications. RNA was extracted from dissected tissues using RNAzol-B (Tel-Test Inc., Friendswood, Texas, USA) according to standard protocol. After isopropanol precipitation, the RNA was resuspended in 0.5 $\mathrm{ml} 0.5 \%$ SDS in diethyl chlorophosphate-treated water and extracted with two volumes of phenol/chloroform, followed by precipitation with sodium acetate and two volumes of ethanol. Samples were left at $-20^{\circ} \mathrm{C}$ overnight and then pelleted. Samples were then resuspended in 0.5 $\mathrm{ml}$ of diethyl chlorophosphate-treated water (except for bone marrow, which was resuspended in $0.1 \mathrm{ml}$ ), and their OD was measured for quantitative and qualitative analysis. Aliquots of $2 \mu \mathrm{g}$ of RNA were treated with $1 \mu \mathrm{l}$ each of RNasin (Promega Corp., Madison, Wisconsin, USA) and $1 \mu$ l DNase I (Invitrogen Corp., Carlsbad, California, USA) and left at room temperature for $15 \mathrm{~min}$ utes, followed by heat denaturation of the DNase I at $90^{\circ} \mathrm{C}$ for 10 minutes. Reverse transcription was performed with AMV-RT (Promega Corp.) according to the standard protocol adjusted to a $30-\mu l$ volume. Oligo $d(T)$ (Applied Biosystems, Foster City, California, USA) was used to prime the reaction at $42^{\circ} \mathrm{C}$ for 1 hour. PCR was carried out on $2-\mu$ l aliquots of the cDNA using Taq polymerase (Roche Applied Science, Basel, Switzerland) in standard buffers for 50 cycles of $95^{\circ} \mathrm{C}$ for 1 minute, $58^{\circ} \mathrm{C}$ for 1 minute, and $72^{\circ} \mathrm{C}$ for 1 minute, followed by $72^{\circ} \mathrm{C}$ for 7 minutes to complete the last cycle. Products were run in $1.5 \%$ agarose in TAE buffer with a 100 -bp ladder size standard (Invitrogen Corp.). FSP1.GFP primers were FSP1, 5' (GTGATTTGGG TCATGTCAG) and GFP 3' (GAACAGCTCCTCGCCC TTGC); GAPDH primers were GAPDH 5' (GCAGTGGCAAAGTGGAGATT) and GAPDH 3' (GCAGAAGGGGCGGAGATGAT).

Unilateral ureteral obstruction. Male and female transgenic mice and wild-type littermates weighing 22-25 g were anesthetized with methoxyflurane following protocols approved by the IACUC at the University of Pennsylvania and Vanderbilt University. After induction of anesthesia, the right flank was opened through a small incision, and the right ureter was completely ligated with 2-0 suture at two points (8). Mice were monitored postoperatively, and both kidneys were harvested for immunohistochemistry 10 days after initial surgery.

Bone marrow chimeras. Bone marrow chimeras were prepared as previously described $(7,8)$. Recipient $\mathrm{Balb} / \mathrm{c}$ mice were treated with antibiotics for 1 week prior to marrow transfer and then irradiated with two doses of 5 Gy 4 hours apart in a cesium 137 irradiator followed by an additional week of antibiotics. Bone marrow cells were isolated from donor long bones by aspiration and flushing, separated from the stroma by gravity, and treated with guinea pig complement plus anti-Thy 1.2 (J1j) antibody to deplete the marrow of donor T cells. Residual donor cells $\left(2 \times 10^{7}\right)$ in $200 \mu \mathrm{l}$ of PBS were injected into the tail vein of irradiated recipients. Some cells were washed and fixed in $1 \%$ formalin and used for FACS scan analyses. Mice were allowed to recover for 30 days. Chimeric protocols were approved by the IACUC Vanderbilt University.
Immunohistochemistry. Immunohistochemistry was performed on paraffin-embedded tissues using standard techniques at $37^{\circ} \mathrm{C}$. Primary antibodies were incubated for 1 hour and secondary antibodies for 30 minutes $(7,8)$. PBS-Tween 20 was added wherever PBS was used, and in staining reactions for FSP1, an unmasking reagent (Vector Laboratories Inc., Burlingame, California, USA) was used in the prestain. Confocal microscopy was performed using either a Zeiss LSM 410 Laser-Scanning Confocal Microscope with $488 \mathrm{~nm}$ excitation for FITC and $543 \mathrm{~nm}$ or 568 $\mathrm{nm}$ excitation for Texas Red (Vanderbilt Cell Imaging Core), or an Olympus Provis microscope (Olympus America, Melville, New York, USA) with an Optronics MagnaFire imaging system (Optronics, Goleta, California, USA) using U-M41001-FITC and U-M41002TRITC filters, at the Vanderbilt Cardiovascular Histology and Imaging Core. Images were taken at magnifications of 400-630×. All images were processed using Photoshop 6.0 (Adobe Systems Inc., San Jose, California, USA) and Canvas 7.0 (Deneba Systems Inc., Miami, Florida, USA). Antibodies for immunohistochemistry were: anti-FSP1 rabbit polyclonal antibody, 1:1,000 (7, 8); FITC- or P6-labeled goat anti-rabbit IgG (Vector Laboratories Inc.), 1:500; anti- $\beta$-gal biotinylated monoclonal antibody (Sigma-Aldrich, St. Louis, Missouri, USA), 1:500; Texas Red avidin D (Vector Laboratories Inc.), 1:300; anti-proliferating cell nuclear antigen goat polyclonal antibody (Santa Cruz Biotechnology Inc., Santa Cruz, California, USA), 1:200; FITC goat anti-rabbit IgG (Vector Laboratories Inc.), 1:500; FITC donkey anti-goat IgG (Jackson ImmunoResearch Laboratories Inc., West Grove, Pennsylvania, USA), 1:500; Texas Red donkey anti-goat IgG (Jackson ImmunoResearch Laboratories Inc.), 1:500; Texas Red goat anti-rabbit IgG (Vector Laboratories Inc.), 1:500; anti-caspase-3 rabbit polyclonal antibody (Santa Cruz Biotechnology Inc.), 1:200; Texas Red goat anti-rabbit IgG (Vector Laboratories Inc.), 1:500; anti-HSP47 goat polyclonal antibody (Santa Cruz Biotechnology Inc.), 1:200; and FITC-conjugated anti-Cre recombinase monoclonal antibody (Covance Research Products, Denver, Pennsylvania, USA), 1:100. In selected experiments, 40-80 random high-powered fields from several cortical kidneys were examined for counts of FSP1 ${ }^{+}$ cells and $\mathrm{LacZ}^{+}$and $\mathrm{GFP}^{+}$fibroblasts in various experimental groups. FACS scans were performed on Becton Dickinson FACS Scan and analyzed with Cell Quest (Becton Dickinson, Franklin Lakes, New Jersey, USA).

Statistics. ANOVA using the Student $t$ test was used to identify significant differences. $P<0.05$ was accepted as significant.

\section{Results}

Demonstration of EMT-derived fibroblasts in fibrotic renal tissue. To test for the role of EMT in the in situ formation of fibroblasts in epithelial tissue, we marked proximal epithelium in the kidneys of R26R mice (29), in which the LacZ reporter gene is separated 


\section{Figure 1}

Characterization of $\gamma$ GT.Cre mice. (a) Plasmid map of the $\gamma \mathrm{GT}$.Cre transgene injected into B $6 \times$ SJL zygotes for the production of transgenic mice. (b) $\gamma \mathrm{GT}$.Cre transgenic mice express Cre transcripts only in the kidney and after postpartum day 7 (day P7), shown by Northern blot probed with cDNA encoding $\mathrm{Cre}$ and GAPDH. B, day of birth. (c) Immunofluorescent staining of a paraffin-embedded kidney section from $\gamma$ GT.Cre transgenic mice using antibody against $\mathrm{Cre}$ demonstrates staining in cortical proximal tubules but not in the medulla by confocal microscopy $(\times 400)$; inset at higher power shows cortical tubular (CT) staining (×630). (d) R26R × yGT.Cre F1 hybrid mice express a recombination amplicon of approximately 575 bp's (shown by PCR) $(7,8)$ in the kidney but not in the liver. bpA, poly A tail; Br, brain; Lu, lung; Ad, adrenal gland; Lv, liver; Sp, spleen; Mu, muscle; Kd, kidney.

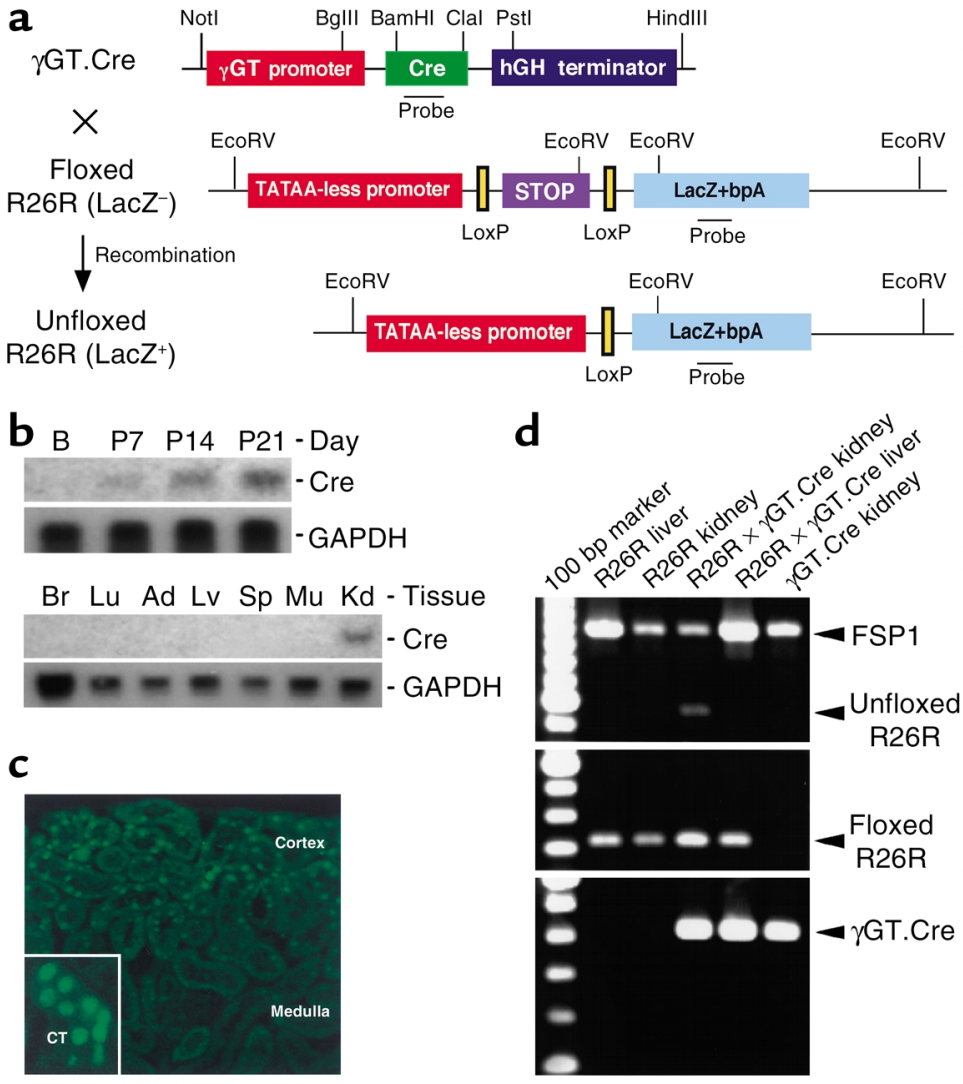

from the ubiquitously expressed Rosa 26 promoter by a floxed STOP (pgk.NEO) sequence (LacZ-), by crossing them with mice transgenic for $\gamma$ GT.Cre recombinase (26) (Figure 1a). The Cre recombinase splices out the STOP sequence in selected cells (unfloxed or $\mathrm{LacZ}^{+}$) in R26R mice, depending on which cells the $\gamma G T$ promoter is active. Our Northern blot experiments $(7,8)$ showed that the $\gamma G T$ promoter expresses transcripts encoding Cre recombinase only in cortical kidneys, not in brain, liver, spleen, muscle, lung, or adrenal gland (Figure 1b). This expression occurs in late kidney development, beginning around postpartum day 14 when nephrogenesis is virtually complete (Figure 1b). Additionally, bone marrow cells did not express $\gamma$ GT.Cre by immunofluorescence (staining was at background levels). Only cortical tubular epithelium from $\gamma$ GT.Cre kidney stains with fluorescent antibody against Cre protein (Figure 1c); other tissues subjected to Northern blot did not stain for $\gamma \mathrm{GT}$ (data not shown). The recombination event predicted that when $\gamma$ GT.Cre mice are crossed with R26R mice, a PCR amplicon is produced in kidney that is not found in liver (Figure 1d). The timing of $\gamma$ GT.Cre promoter activity permits cortical tubular epithelium to be selectively marked only in mature renal tissue.

Eight weeks after birth, R26R $\times \gamma$ GT.Cre F1 mice underwent a surgical procedure to create unilateral ureteral obstruction (UUO) producing experimental renal fibrosis in one kidney $(8,30)$. Progressive interstitial fibrosis following an inflammatory reaction in the obstructed kidney is diffuse, becomes evident histologically 10 days after surgery, and leads to complete destruction of the kidney after 3-4 weeks; chemical renal failure, however, does not ensue because the unobstructed, contralateral kidney remains normal and supports glomerular filtration (31). Day 10 after UUO was used to illustrate our experimental findings because early fibrogenesis was demonstrable at this time, but anatomical landmarks were still identifiable. At that time both kidneys were harvested and embedded in paraffin. Histologic sections were cut and stained with fluorescent antibodies against FSP1 (green) and LacZ (red) and examined by confocal microscopy.

Confocal microscopy of the normal kidneys from $\mathrm{R} 26 \mathrm{R} \times \gamma \mathrm{GT}$.Cre F1 mice 10 days after unilateral obstruction showed unfloxed FSP1-, $\mathrm{LacZ}^{+}$cortical tubular epithelium stained red $\left(\mathrm{LacZ}^{+}\right)$and floxed $\mathrm{FSP}^{+}$, LacZ- interstitial fibroblasts stained green $\left(\mathrm{LacZ}^{-}\right)$, reflecting that those fibroblasts formed prior to the postnatal activation of $\gamma$ GT.Cre; glomeruli did not stain (Figure 2a). There were no merged signals among interstitial cells to suggest EMT activity in the normal contralateral kidney at the time of tissue acquisition. Obstructed and fibrotic kidneys from these mice, on the other hand, revealed a mixture of epithelial and interstitial cell reaction products amidst this injured tissue. As shown in Figure 2b, representative FSP1 ${ }^{+} \mathrm{LacZ}^{+}$ tubular epithelium undergoing disaggregation in an obstructed kidney 10 days after UUO stained yellow on merged color under confocal microscopy. These $\mathrm{LacZ}^{+}$ 
epithelial cells were $\mathrm{FSP} 1^{+}$as evidence of transition during EMT (7); some of these transitional epithelial cells also began to take the shape of FSP1 ${ }^{+}, \mathrm{LacZ}^{+}$fibroblasts. As shown in Figure 2, c and d, floxed FSP1 ${ }^{+}$, LacZ fibroblasts 10 days after UUO stained green, while newly formed, unfloxed $\mathrm{FSP}^{+}$, $\mathrm{LacZ}^{+}$fibroblasts (double positive for FSP1 and LacZ) stained yellow, indicating that the fibrogenic process does not activate $\gamma$ GT.Cre recombinase in fibroblasts; some FSP1 ${ }^{+}, \mathrm{LacZ}^{+}$ cortical tubular epithelium undergoing EMT, again, merged yellow as well.

Because it is difficult to directly determine which cells in a fibrotic reaction contribute to interstitial collagen production by measuring intracellular collagen synthesis in vivo, we used the surrogate marker HSP47, which abundantly increases to chaperon type I collagen molecules in cells actively engaged in collagen synthesis $(30,32)$. Since the fibrogenesis following UUO is associated with increased deposition of interstitial collagen (30), UUO kidneys from R26R × $\gamma$ GT.Cre F1 mice were examined after 10 days for evidence that unfloxed $\mathrm{LacZ}^{+}$tubular epithelium and $\mathrm{LacZ}^{+}$fibroblasts in fibrotic renal tissue contributed to interstitial collagen production. Double staining for HSP47 (green) (Figure $2 \mathrm{e}$ ) and LacZ (red) (Figure 2f) developed yellow on merged color (Figure $2 \mathrm{~g}$ ) in $\mathrm{LacZ}^{+}, \mathrm{HSP}_{4} 7^{+}$tubular epithelium and in surrounding $\mathrm{LacZ}^{+}, \mathrm{HSP} 47^{+}$fibrob- lasts, indicating that both populations of cells during EMT contribute collagen to the fibrogenic response. FSP1 $1^{+}$fibroblasts migrate from bone marrow to fibrotic kidney. We next determined whether peripheral, non-kidney fibroblasts migrate to the renal interstitium from external sites (6). To do this, we first marked fibroblasts in transgenic mice with GFP under the control of the fibroblast-specific FSP1 promoter (Figure 3a). Mouse line M10, carrying approximately eight copies of the transgene FSP1.GFP, was among several providing a green skin color at birth under a Woods light (not shown) and green eyes (corneas) in adults (Figure 3b). RT-PCR amplification of GFP sequences from normal organ tissues revealed a variable amount of FSP1 expression from whole-organ mRNA (Figure 3c). This variability in mRNA encoding FSP1 is consistent with the disparity in the number of fibroblasts found in different normal tissues; few in liver, kidney, and bone marrow, and more in skin, thymus, heart/pericardium, and brain (probably astrocytes). Costaining of $\mathrm{GFP}^{+}$ fibroblasts with anti-FSP1 antibody (red) resulted in double-labeled cells in representative samples of kidney, liver, heart, and lungs (Figure $3 \mathrm{~d}$ ), suggesting (as observed before, ref. 8) that the FSP1 genomic fragment used as the promoter selectively operates in fibroblasts. In normal kidneys from adult FSP1.GFP mice, green $\mathrm{FSP}^{+}, \mathrm{GFP}^{+}$fibroblasts were observed occasionally in

\footnotetext{
Figure 2

Confocal microscopy images of merged stainings from kidney tissue following UUO. (a) Glomeruli from the contralateral kidneys in R26R $\times \gamma G T$.Cre mice 10 days after unilateral UUO have no reaction product. Unfloxed LacZ $Z^{+}$tubular epithelium stained red with anti-LacZ antibodies as evidence of recombination, and floxed LacZ- interstitial fibroblasts formed before day P7 stained green (FSP1+) with antiFSP1 antibodies. $\times 630$. (b) A representative cortical tubule undergoing EMT in kidney harvested 10 days after UUO stained with anti-FSP1 (green) and anti-LacZ (red). The merged confocal image demonstrates unfloxed $\mathrm{FSP}^{+}$, $\mathrm{LacZ}^{+}$epithelial cells staining yellow as double-positive cells. The tubule is disaggregating, with cellular elements assuming the shape of new interstitial FSP1 ${ }^{+}$, LacZ ${ }^{+}$fibroblasts. $\times 630$. (c) Renal cortical interstitium from kidney harvested 10 days after UUO demonstrated that floxed FSP1 ${ }^{+}$, LacZ $\mathrm{L}^{-}$fibroblasts formed before day $\mathrm{P7}$ were stained green $\left(\mathrm{FSP} 1^{+}\right)$, and adjacent and newly formed unfloxed FSP1 ${ }^{+}$, LacZ ${ }^{+}$fibroblasts (those created after day P7) stained yellow (double positive for FSP1 and LacZ) after local EMT. $\times 400$. (d) Tubular EMT in kidney cortical tissue harvested 10 days after UUO demonstrated that floxed FSP1 ${ }^{+}$, LacZ ${ }^{-}$fibroblasts formed before day $\mathrm{P} 7$ were stained green $\left(\mathrm{FSP} 1^{+}\right)$, some cortical $\mathrm{FSP}^{+}$, $\mathrm{LacZ}^{+}$tubular epithelial cells undergoing EMT were yellow (double positive for FSP1 and LacZ), and adjacent and newly formed, unfloxed $\mathrm{FSP}^{+}$, LacZ ${ }^{+}$fibroblasts stained yellow after EMT. $\times 630$. (e) Day 10 UUO kidney sections stained with anti-LacZ (green) and (f) anti-HSP47 (red). (g) Merged kidney section in yellow demonstrates colocalization of HSP47 (collagen type I production) in unfloxed tubular $\mathrm{LacZ}^{+}, \mathrm{HSP} 47^{+}$epithelium undergoing EMT and in new unfloxed LacZ ${ }^{+}, \mathrm{HSP} 47^{+}$fibroblasts. $\times 400$.
}

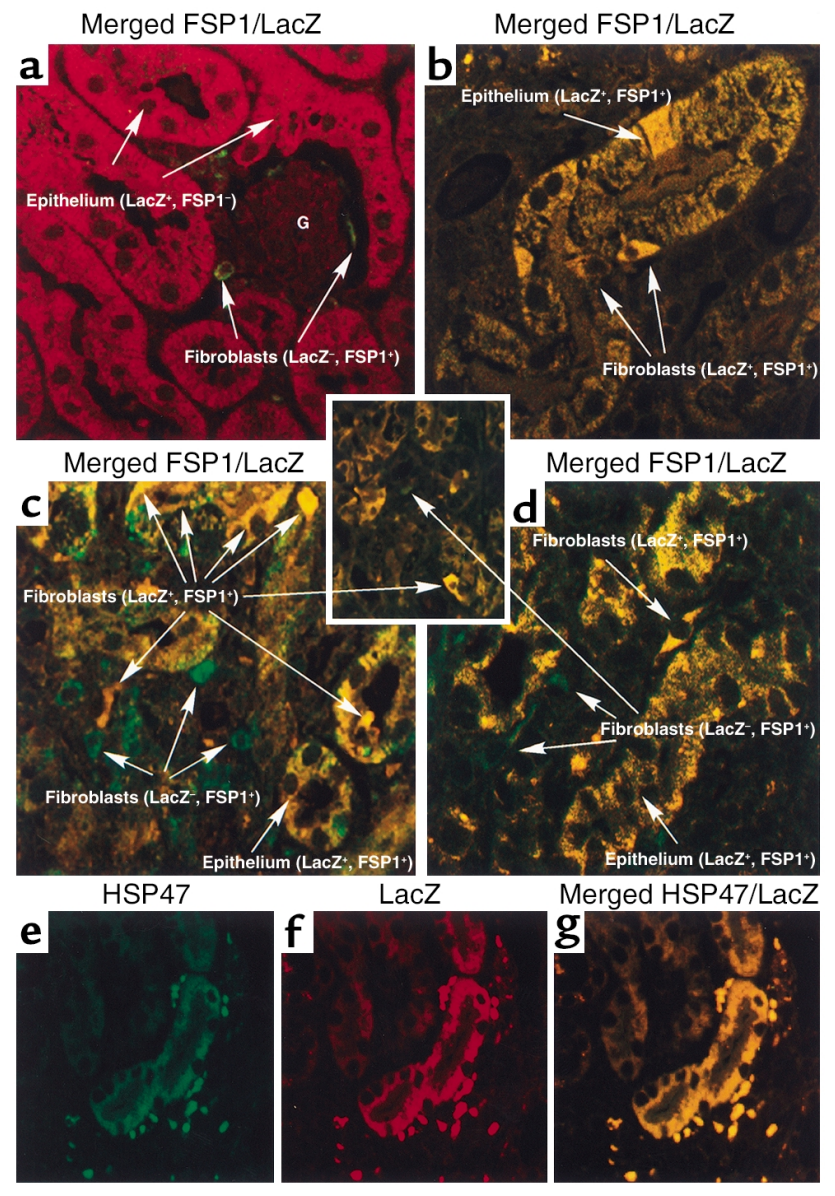



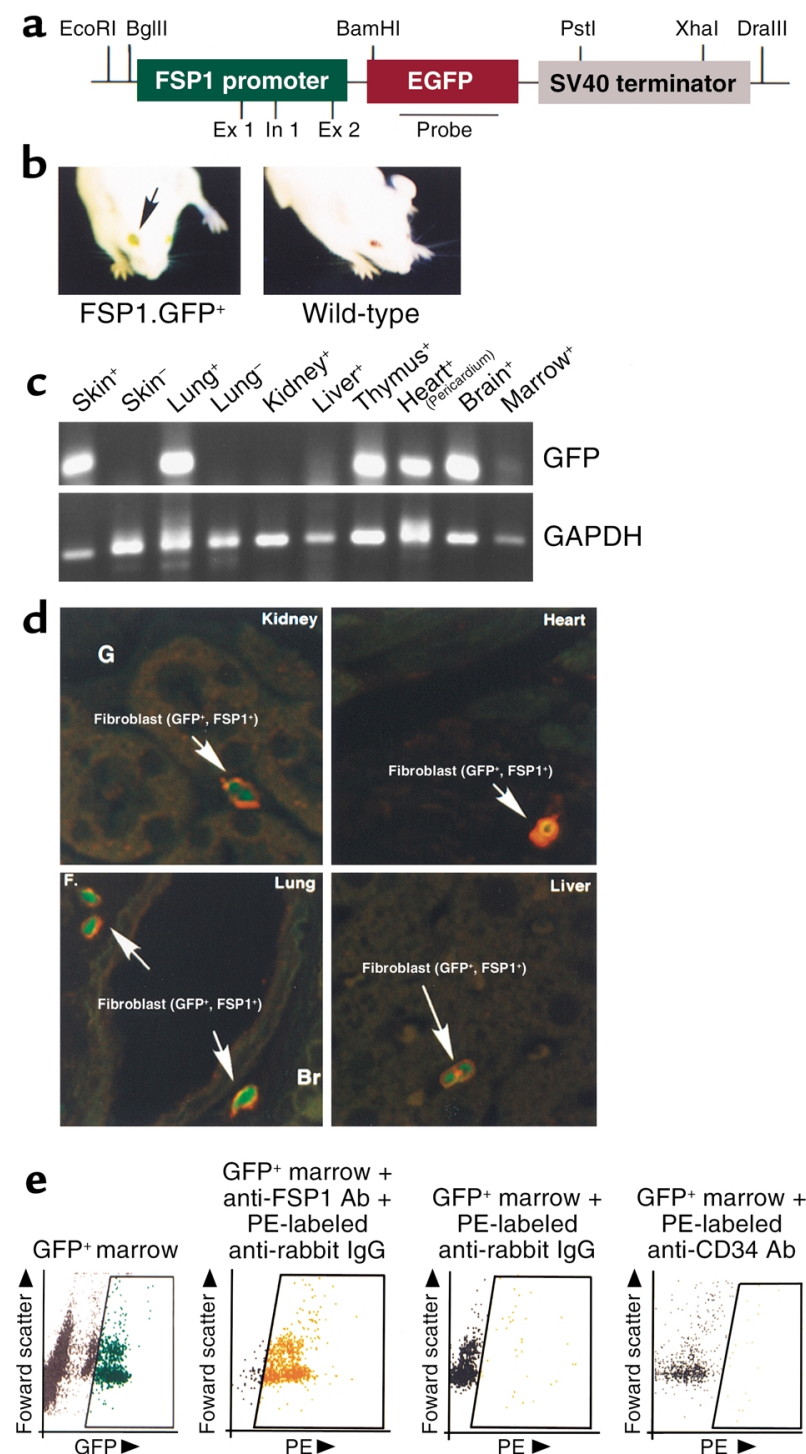

$\mathrm{GFP}^{+}$marrow + anti-FSP1 $\mathrm{Ab}+\mathrm{GFP}^{+}$marrow + PE-labeled PE-labeled anti-rabbit IgG
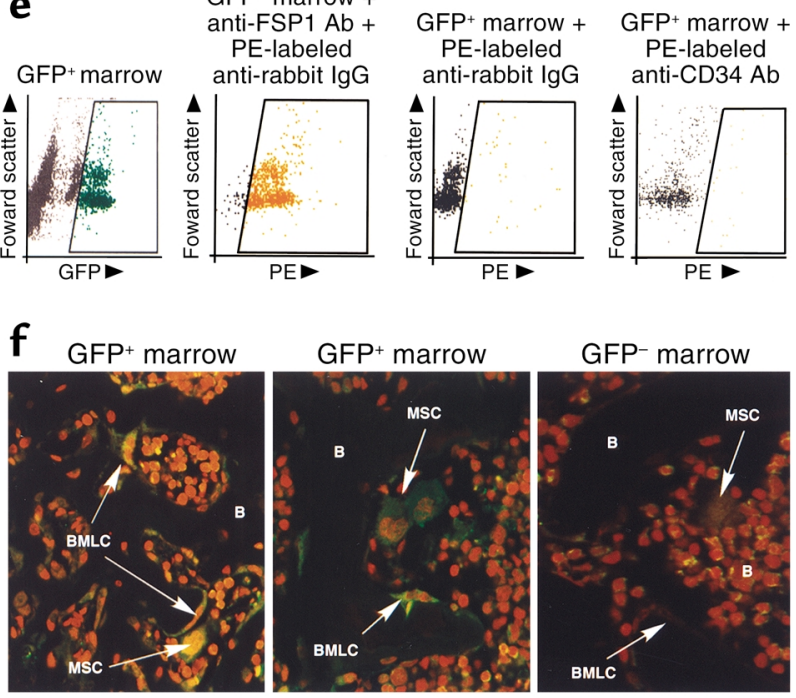

the interstitial spaces between tubules, suggesting that resident fibroblasts are rarely found in this location (7). A FACScan of bone marrow cells from the femurs of these mice demonstrated that 5-6\% of marrow cells were fluorescent green (Figure 3e) and that nearly all of these cells costained with anti-FSP1 antibody; furthermore, less than $1 \%$ of these $\mathrm{GFP}^{+}$fibroblasts were $\mathrm{CD}^{4+}$; preliminary evidence suggests that $\mathrm{FSP}^{+}$, CD34- bone marrow cells contain several subpopulations: Gr-1+ $1^{+}(79 \%), \mathrm{Flk}^{+}(15 \%), \mathrm{Sca}^{+}{ }^{+}(10 \%), \mathrm{Kit}^{+}(4 \%)$, and Thy- $1^{+}(<1 \%)$; data not shown.

\section{Figure 3}

Characterization of FSP1.GFP transgenic mice. (a) Plasmid map of the FSP1.GFP transgene injected into B6D2 zygotes for the production of transgenic mice. Ex 1, exon 1; In 1, intron 1. (b) Adult FSP1.GFP transgenic mice express GFP protein in all tissue fibroblasts. In adult FSP1.GFP mice, the eyes are demonstrably green under a Woods light compared with the wild-type because the $\mathrm{GFP}^{+}$fibroblasts in the cornea cast a green tint. (c) RT-PCR screen of normal, whole-organ tissues for mRNA encoding GFP from $\mathrm{GFP}^{+}(+)$or GFP- $(-)$littermates: skin, lungs, liver, kidney, heart/pericardium, thymus, and brain. (d) Green $\mathrm{GFP}^{+}$fibroblasts from normal FSP1.GFP kidneys, liver, lung, and heart that costained with anti-FSP1 antibody (red) in their cytoplasm were occasionally observed in interstitial organ spaces; between tubules in kidney. $\times 630$. (e) FACScan of FSP1.GFP bone marrow shows that $5-6 \%$ of FSP $1^{+}$marrow cells are $\mathrm{GFP}^{+}$and that $95 \%$ of these $\mathrm{FSP}^{+}, \mathrm{GFP}^{+}$fibroblasts costain with anti-FSP1 antibody labeled with phycoerythrin (PE). Approximately $95 \%$ of $\mathrm{GFP}^{+}$fibroblasts were also CD34-. (f) Decalcified bones from FSP1.GFP and GFP- wild-type mice were costained with propidium iodide, and $\mathrm{GFP}^{+}$cells were identified in two locations by confocal microscopy, either scattered throughout the abluminal marrow as $\mathrm{FSP} 1^{+}, \mathrm{MSC}$ s tethered to the sinusoidal network, or as FSP1 ${ }^{+}, \mathrm{GFP}^{+}$endosteal lining cells (BMLCs) adjacent to decalcified osteoid (B). $\times 630$.

Confocal microscopy of decalcified bone counterstained with propidium iodide identified these $\mathrm{FSP} 1^{+}$, CD34- cells in two locations (Figure 3f). Some GFP' MSCs were scattered along the abluminal marrow, seemingly tethered to a sinusoidal network surrounded by hematopoietic cells. The other site where these $\mathrm{GFP}^{+}$cells were identified was along the shaft and trabecular endosteum as adherent bone marrow lining cells (BMLCs). It is possible that the MSCs identified were actually BMLCs caught on a confocal plane capturing a rear osteoid surface. More likely in our opinion is that the cells identified as MSCs in Figure 3 f are the $\mathrm{GFP}^{+}$cells seen on FACScan in Figure $3 \mathrm{e}$.

After seven generations of backcrossing to wild-type $\mathrm{Balb} / \mathrm{c}$ mice, $\mathrm{T}$ cell-depleted marrow from FSP1.GFP $\mathrm{Balb} / \mathrm{c}$ transgenic mice was intravenously transferred into lethally irradiated (10 Gy) wild-type (GFP-) Balb/c recipients (33). (T cells were removed from the donor marrow to obviate any potential graft-versus-host disease.) Thirty days later, chimeric mice underwent UUO, and kidneys and bone marrow were harvested 10 days after that. Donor bone marrow from FSP1.GFP mice containing green $\mathrm{FSP}^{+}, \mathrm{GFP}^{+}$fibroblasts was compared with marrow from chimeric recipients collected just after UUO (Figure 4a). Bone marrow profiles of donor mice and chimeric recipients produced by FACScan showed that green FSP1 $1^{+}, \mathrm{GFP}^{+}$donor MSC successfully repopulated the bone marrow of chimeric recipients. Furthermore, $\mathrm{FSP}^{+}, \mathrm{GFP}^{+}$donor fibroblasts in these chimeras were observed in obstructed kidney tissue 10 days after UUO, but were rarely found in normal contralateral kidneys (Figure 4b). Some but not all of these interstitial FSP $1^{+}, \mathrm{GFP}^{+}$fibroblasts, obtained from both donor and recipient kidneys following UUO, costained with HSP47 (Figure 4c), indicating they also contribute interstitial collagens to the fibrogenic process. 
Assessment of the relative contributions of bone marrow and EMT to fibroblast production. Quantitation of FSP1 ${ }^{+}$ fibroblasts in kidneys subjected to UUO and normal contralateral kidneys after tubular EMT, or after migration from bone marrow in chimeric recipients, was performed by counting multiple random cortical fields from several different conditions. $\mathrm{FSP}^{+}, \mathrm{GFP}^{+}$bone marrow fibroblasts contribute no more than $12 \%$ of the expected resident fibroblasts in normal tissue (number of $\mathrm{FSP}^{+}, \mathrm{GFP}^{+}$fibroblasts from normal, chimeric recipient kidneys divided by the number of $\mathrm{FSP}^{+}, \mathrm{GFP}^{+}$ fibroblasts from FSP1.GFP ${ }^{+}$donor kidneys; Figure 5). A review of normal contralateral kidneys from $\mathrm{R} 26 \mathrm{R} \times \gamma \mathrm{GT}$.Cre mice following UUO failed to demonstrate any spontaneous EMT-derived $\mathrm{FSP}^{+}$, $\mathrm{LacZ}^{+}$ fibroblasts in adult kidney tissue in the absence of a fibrogenic stimulus (number of $\mathrm{FSP}^{+}{ }^{+} \mathrm{LacZ}^{+}$fibroblasts from normal kidneys divided by the number of FSP $1^{+}$fibroblasts from normal kidneys; Figure 5). During fibrogenesis, however, the portion of $\mathrm{FSP}^{+}, \mathrm{LacZ}^{+}$fibroblasts contributed by EMT in R26R $\times \gamma$ GT.Cre mice rose to $36 \%$ of the $\mathrm{FSP}^{+}$interstitial cells (number of $\mathrm{FSP}^{+}$, $\mathrm{LacZ}^{+}$ fibroblasts from UUO kidneys divided by the number of FSP1 ${ }^{+}$fibroblasts from UUO kidneys; Figure 5).
The $\mathrm{FSP}^{+}, \mathrm{GFP}^{+}$fibroblasts migrating to fibrogenic kidney from bone marrow reconstituted with FSP1.GFP donor cells contributed $15 \%$ of the FSP1 ${ }^{+}$ fibroblasts in the interstitium, $3 \%$ more than proportionally found in the normal contralateral kidney (number of $\mathrm{FSP}^{+}, \mathrm{GFP}^{+}$fibroblasts from UUO chimeric recipient kidneys divided by the number of $\mathrm{FSP}^{+}$fibroblasts from UUO kidneys; Figure 5), although the absolute numbers of both EMT-derived $\mathrm{LacZ}^{+}$and bone marrow-derived $\mathrm{GFP}^{+}$populations of $\mathrm{FSP}^{+}$fibroblasts were increased in fibrogenic kidneys. Double staining for proliferating cell nuclear antigen (34) and FSP1 during renal fibrogenesis 10 days after UUO also demonstrated that $42 \%$ of proliferating interstitial FSP1+ fibroblasts carried the EMT marker LacZ, but only $27 \%$ of proliferating fibroblasts originated from $\mathrm{GFP}^{+}$bone marrow (data not shown), suggesting that different rates of proliferation over time might skew the number of local and bone marrow-derived fibroblasts in favor of those $\mathrm{FSP}^{+}$, $\mathrm{LacZ}^{+}$fibroblasts arising from EMT.

Finally, we speculate that any remaining populations of fibroblasts not LacZ-marked as cortical tubules or arising from $\mathrm{GFP}^{+}$bone marrow likely result from EMT
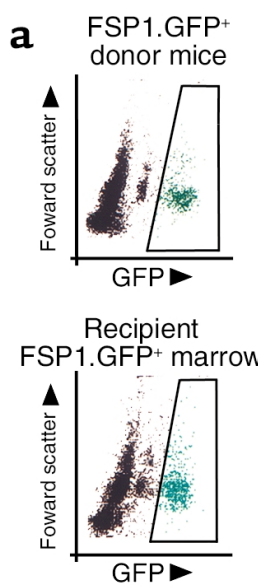

Donor FSP1.GFP- marrow

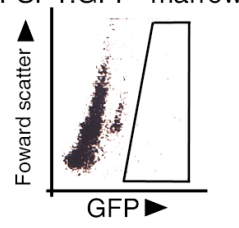

b Recipient FSP1.GFP+ marrow

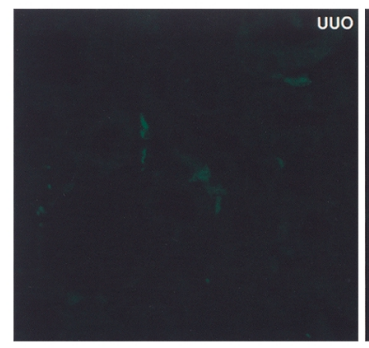

Recipient FSP1.GFP ${ }^{+}$ marrow

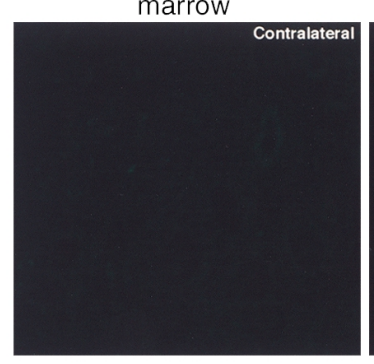

C Merged FSP1.GFP $+/ \mathrm{HSP}_{4} 7^{+}$ FSP1.GFP+ donor mice
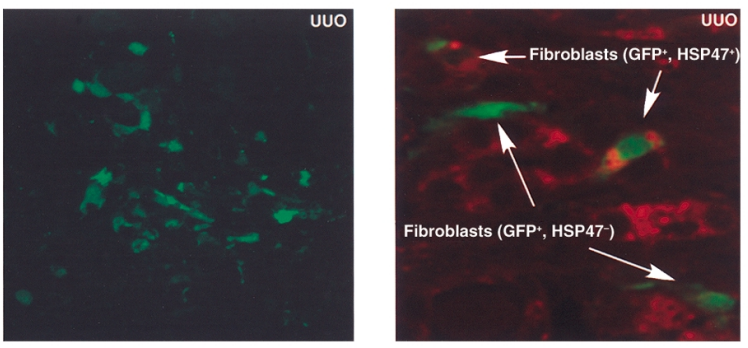

Merged FSP1.GFP $+/ \mathrm{HSP} 47^{+}$ recipient marrow

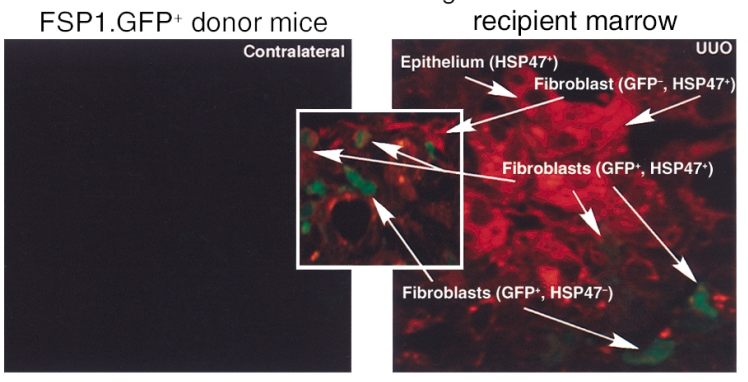

Figure 4

Bone marrow chimeras were produced by transfer of bone marrow from FSP1.GFP transgenic mice to Balb/c wild-type mice. After 30 days of recovery, they underwent surgical UUO; kidney and bone marrow were harvested 10 days later. (a) GFP ${ }^{+}$donor marrow containing FSP1 ${ }^{+}$ stromal fibroblasts (green) and representing 5-6\% of the donor cell population on FACScan were found in chimeric recipients at harvest 10 days after UUO in approximately the same proportion as in wild-type donor marrow where there was no GFP expression. (b) Tissues harvested from chimeric mice following UUO of one kidney demonstrate a rare green $\mathrm{FSP}^{+}$, $\mathrm{GFP}^{+}$fibroblast in the interstitium of the normal, contralateral control kidney. More fibroblasts of this type appear in the kidney stressed by UUO and fibrogenesis $\times 400$. FSP1.GFP transgenic mice were also subjected to UUO in parallel. These UUO kidneys also showed an increase in green $\mathrm{FSP}^{+}$, $\mathrm{GFP}^{+}$fibroblasts compared with the contralateral control (data not shown). (c) Merged confocal microscopy of kidney sections 10 days after UUO in donor or recipient mice demonstrates a mixture of GFP+, HSP47- fibroblasts (GFP shows as green), GFP-, HSP47 ${ }^{+}$fibroblasts (HSP47 shows as red), and doublelabeled $\mathrm{GFP}^{+}, \mathrm{HSP} 47^{+}$fibroblasts (green/red or weak yellow). GFP-, HSP47 ${ }^{+}$tubular epithelial cells from UUO kidney tissue also stained red, reflecting their contribution to collagen type I production during EMT. $\times 630$. 


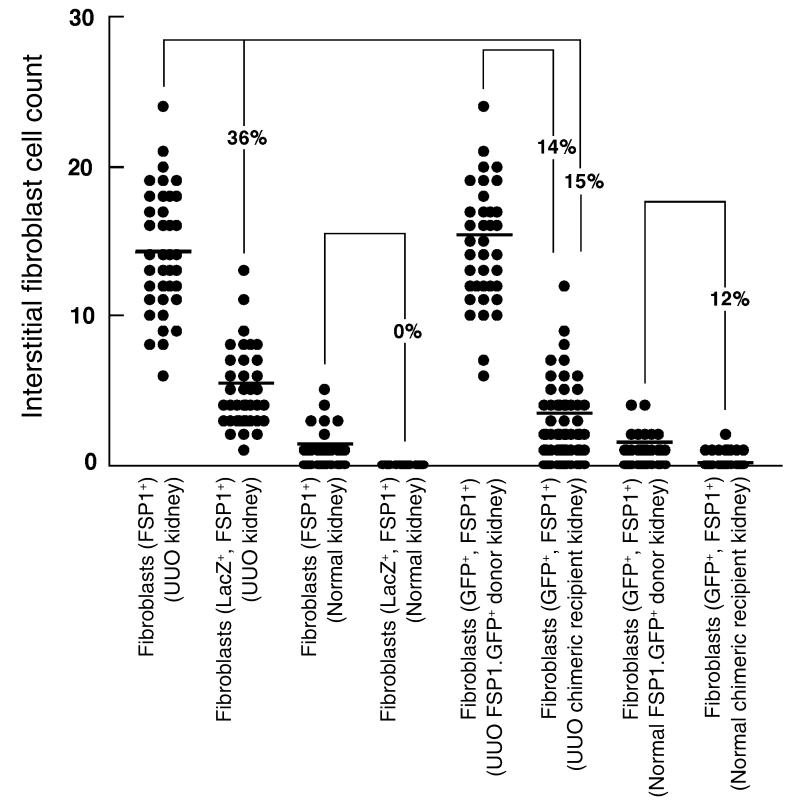

Figure 5

Fibroblast cell counts in renal tissues after UUO (8). Several different groups of kidneys were compared to determine the relative contributions of each to the source of fibroblasts. Forty to 80 random high-power fields in cortical kidneys were counted for FSP1 ${ }^{+}$fibroblasts, EMT-derived $\mathrm{LacZ}^{+}$fibroblasts, or $\mathrm{GFP}^{+}$fibroblasts, depending on the group. Mean fibroblast counts are represented by a horizontal line in each column: $12 \%$ of normal resident fibroblasts in the kidney come from bone marrow (number of FSP1 ${ }^{+}$, $\mathrm{GFP}^{+}$fibroblasts from normal, chimeric recipient kidneys divided by the number of FSP1 ${ }^{+}, \mathrm{GFP}^{+}$fibroblasts from FSP1.GFP ${ }^{+}$donor kidneys); local EMT as a source is rare in the absence of fibrogenic stress (number of $\mathrm{FSP}^{+}{ }^{+} \mathrm{LacZ}^{+}$fibroblasts from normal kidneys divided by the number of FSP1 ${ }^{+}$fibroblasts from normal kidneys); and during experimental fibrosis, local EMT (number of FSP1 ${ }^{+}$, LacZ $\mathrm{L}^{+}$fibroblasts from UUO kidneys divided by the number of FSP1 ${ }^{+}$fibroblasts from UUO kidneys) and bone marrow (number of FSP1 ${ }^{+}, \mathrm{GFP}^{+}$ fibroblasts from UUO, chimeric recipient kidneys divided by the number of FSP1 ${ }^{+}$fibroblasts from UUO kidneys) contribute $36 \%$ and $15 \%$ of fibroblasts, respectively.

in other unmarked tubular segments affected by fibrogenesis and/or from the proliferation of resident fibroblasts formed prior to injury.

\section{Discussion}

Our results provide proof of principle that fibroblasts can form locally by EMT during the pathologic stress of tissue fibrosis. We prefer the term epithelial-mesenchymal transition (EMT) to describe this conversion instead of epithelial-mesenchymal transdifferentiation because transdifferentiation conventionally refers to one type of epithelium changing into a different epithelium (35). It is not entirely clear whether the mesenchymal transition of EMT is an expected middle phase of transdifferentiating epithelium, whether EMT producing fibroblasts is an arrested form of transdifferentiation, or whether they are really different processes and not just a timeline issue. Epithelial cells that disaggre- gate from their functional units lose their morphogenic cues and "select" new fates, which are probably determined by local cytokines found in the transitional environment. Epithelia that are disaggregating have several phenotypic choices; some may undergo apoptosis, enter into EMT and secrete interstitial collagens, or reside and/or divide as fibroblasts. We have observed all these phenotypes in some of the marked cortical tubular epithelial cells reported on here (caspase- 3 and proliferating cell nuclear antigen staining were not shown).

The process of replenishing fibroblasts from epithelium in adult tissues has many parallels to the conversion of primary and secondary epithelium to mesenchymal cells during various stages of early embryologic development. For example, EMT is seen in the formation of mesoderm during gastrulation (15), with closure of the midline palate (36), and in the formation of connective tissue cells from somitic epithelium (37). Since FSP1, a distinct marker of fibroblasts, does not appear in any cells from the mouse embryo until day E9, and is not found in early mesenchymal cells (7), we believe its relatively late appearance suggests that $\mathrm{FSP}^{+}$fibroblasts found in adult interstitial tissues are not simply residual mesenchymal cells left over from early organogenesis. Accordingly, we advance the hypothesis that fibroblasts in normal organ tissues derive from several sources. Some fibroblasts emerge from a marrow transition, perhaps involving endosteal lining cells in bone marrow (38) that then circulate and reside as a small fraction of interstitial cells. More commonly, however, they appear following local EMT when epithelial tissues are first forming resident fibroblasts and subsequently when epithelia respond to injury.

Most investigators accept the conventional wisdom that fibroblasts represent a cell type of limited diversity. Fibroblast shape, cytoskeletal structure, secretion of interstitial collagens, mobility, participation in tissue fibrosis, and behavior in culture all tend to support this belief. Our EMT hypothesis, however, challenges this notion of homogeneity, as do the observations that fibroblasts express subtle biochemical differences (39) and phenotypic variability (40-42) and respond differently to cytokines and matrix (43), depending on their tissue of origin. Consequently, we suspect that fibroblasts formed by EMT differentially express some residual receptors from their previous life as mature epithelium, and theoretically can be as heterogeneous as the universe of epithelium.

At the level of a single cell, however, this imprinted heterogeneity is less obvious in cultured epithelia that undergo EMT with strong cytokine pressure from signaling moieties like TGF- $\beta(18,44)$, EGF (18), and FGF-2 (22). Most cells under persistent chemical exposure lose their expression of cadherins and substratum adhesion molecules during transition, and begin expressing FSP1, MMP-2, vimentin, type I collagen, and $\alpha$-smooth muscle actin $(18,24,44-46)$. Such uniformity in the expression of potential fibroblast markers in culture is not seen in vivo, and consequently not all 
markers suitably identify fibroblasts in tissue (19). FSP1 expression, as an exception, is found in all resting and activated tissue fibroblasts (7), while vimentin is more unpredictable (19). Type I collagen synthesis is generally detectable only in selected subpopulations of fibroblasts (47-50). Furthermore, only some subpopulations of fibroblasts express $\alpha$-smooth muscle actin (51), a marker of activated fibroblasts (myofibroblasts) $(17,52) . \alpha$-smooth muscle actin is not expressed in all FSP1 ${ }^{+}$fibroblasts (19), suggesting that it either does not define the universe of fibroblasts, or perhaps shares expression with smooth muscle cells separated from local blood vessels in injured tissues $(19,53)$. We currently believe FSP1 is the best, or perhaps the most consistent, marker of fibroblasts in tissue.

Finally, recent studies suggest that mobile progenitors from bone marrow can be induced to replace specialized adult renal cells (mesangial cells, tubular epithelium, and podocytes) $(54,55)$. These conversions may be similar to the mesenchymal-epithelial transition seen in renal embryogenesis (56). However, we did not see any $\mathrm{GFP}^{+}$glomerular or tubular cells in our FSP1.GFP $\rightarrow$ wild-type bone marrow chimeric recipients, suggesting that the source phenotype of our $\mathrm{GFP}^{+}$ marrow cells may uniquely define a progenitor population not susceptible to mesenchymal-epithelial transition, or that local stress is required to complete this transition. Consequently, the expression of FSP1 in endosteal lining cells and MSCs from our FSP1.GFP mice is of interest.

Not much is known about the origin of endosteal lining cells (BMLCs) $(13,57)$. Endosteal lining cells precede the formation of the marrow cavity and its contents (13), and in some species are separated from medullary hematopoiesis by a marrow sac comprising a mixture of simple epithelium and/or condensed stromal-like cells $(58,59)$. This sac appears to have a structural and biochemical interface with endosteal lining cells in nodal regions of bone $(58,60)$, and the collective structure may be an EMT niche for osteogenic precursor cells, indifferent endosteum, and MSCs. It was surprising that most of the $\mathrm{GFP}^{+}$cells in the marrow of FSP1.GFP mice were CD34-. Some observers report that CD34- progenitor cells cycle their expression of CD34 in the marrow (12, $61,62)$, that both CD34- and CD34+ ${ }^{+}$stromal cells circulate in peripheral blood, and following bone marrow reconstitution, CD34- cells locate as bone-lining endosteum $(57,62)$. Since some MSCs can be released into the circulation (62), we speculate that our $\mathrm{FSP}^{+}, \mathrm{CD} 34^{-}$ bone marrow fibroblasts might derive from an endosteal EMT niche transitioning to MSCs, which then may or may not transition to a fibroblast phenotype. As pluripotent progenitors, CD34- marrow cells can form other hematopoietic cells as well as osteoblastic, chondrocytic, and myoblastic lineages $(12,62)$. We are currently evaluating the subset composition and origin of $\mathrm{FSP}^{+}$, CD34- cells in the bone marrow of FSP1.GFP mice.

Progressive tissue fibrosis is the structural equivalent of advancing chemical dysfunction in organs that are failing. The loss of epithelial units, conversion of type IV collagen synthesis to collagen types I and III, and progressive cicatrization are all consistent with the view that EMT is a fundamental part of this pathophysiology. We would not be surprised if other differentiated cells such as endothelium, stellate cells, astrocytes, or myocytes could also transition to fibroblasts when necessitated by the pathologic remodeling of interstitial tissues.

\section{Acknowledgments}

We thank Brigid Hogan and Harold Moses at Vanderbilt University for helpful comments during the early preparation of this manuscript. Part of this work was presented in abstract form at the annual Meeting of the American Society of Nephrology in October, 2000. E.G. Neilson is supported in part by NIH grants DK-46282 and HL-68121.

1. Peifer, M., and McEwen, D.G. 2002. The ballet of morphogenesis: unveiling the hidden choreographers. Cell. 109:271-274.

2. Watt, F.M., and Hogan, B.L.M. 2000. Out of Eden: stem cells and their niches. Science. 287:1427-1430.

3. Spradling, A., Drummond-Barbosa, D., and Kai, T. 2001. Stem cells find their niche. Nature. 414:98-104.

4. Weissman, I.L. 2000. Translating stem and progenitor cell biology to the clinic: barriers and opportunities. Science. 287:1442-1445.

5. Blau, H.M., Brazelton, T.R., and Weimann, J.M. 2001. The evolving concept of a stem cell: entity or function? Cell. 105:829-841.

6. Prockop, D.J. 1997. Marrow stromal cells as stem cells for nonhematopoietic tissues. Science. 276:71-74.

7. Strutz, F., et al. 1995. Identification and characterization of a fibroblast marker: FSP1. J. Cell Biol. 130:393-405.

8. Iwano, M., et al. 2001. Conditional abatement of tissue fibrosis using nucleoside analogs to selectively corrupt DNA replication in transgenic fibroblasts. Mol. Ther. 3:149-159.

9. Bucala, R., Spiegel, L.A., Chesney, J., Hogan, N., and Cerami, A. 1994. Circulating fibrocytes define a new leukocyte subpopulation that mediates tissue repair. Mol. Med. 1:71-81.

10. Friedenstein, A.J., et al. 1974. Precursors for fibroblasts in different populations of hematopoietic cells as detected by the in vitro colony assay method. Exp. Hematol. 2:83-92.

11. Abe, R., Donnelly, S.C., Peng, T., Bucala, R., and Metz, C.N. 2001. Peripheral blood fibrocytes: differentiation pathway and migration to wound sites. J. Immunol. 166:7556-7562.

12. Krause, D.S., et al. 2001. Multi-organ, multi-lineage engraftment by a single bone marrow-derived stem cell. Cell. 105:369-377.

13. Bianco, P., and Robey, P.G. 2000. Marrow stromal cells. J. Clin. Invest. 105:1663-1668.

14. Hay, E.D., and Zuk, A. 1995. Transformations between epithelium and mesenchyme: normal, pathological, and experimentally induced. Am.J. Kidney Dis. 26:678-690.

15. Tam, P.P., and Behringer, R.R. 1997. Mouse gastrulation: the formation of a mammalian body plan. Mech. Dev. 68:3-25.

16. Boyer, B., Valles, A.M., and Edme, N. 2000. Induction and regulation of epithelial-mesenchymal transitions. Biochem. Pharmacol. 60:1091-1099.

17. Ng, Y.Y., et al. 1998. Tubular epithelial-myofibroblast transdifferentiation in progressive tubulointerstitial fibrosis in $5 / 6$ nephrectomized rats. Kidney Int. 54:864-876.

18. Okada, H., Danoff, T.M., Kalluri, R., and Neilson, E.G. 1997. The early role of FSP1 in epithelial-mesenchymal transformation. Am. J. Physiol. 273:563-574.

19. Okada, H., et al. 2000. Progressive renal fibrosis in murine polycystic kidney disease: an immunohistochemical observation. Kidney Int. 58:587-597.

20. Zeisberg, M., et al. 2001. Renal fibrosis: collagen composition and assembly regulates epithelial-mesenchymal transdifferentiation. Am. J. Pathol. 159:1313-1321.

21. Zavadil, J., et al. 2001. Genetic programs of epithelial cell plasticity directed by transforming growth factor-beta. Proc. Natl. Acad. Sci. USA. 98:6686-6691.

22. Strutz, F., et al. 2002. Role of basic fibroblast growth factor- 2 in epithelial-mesenchymal transformation. Kidney Int. 61:1714-1728.

23. Zondag, G.C., et al. 2000. Oncogenic Ras downregulates Rac activity, which leads to increased Rho activity and epithelial-mesenchymal transition. J. Cell Biol. 149:775-782. 
24. Cano, A., et al. 2000. The transcription factor snail controls epithelialmesenchymal transitions by repressing E-cadherin expression. Nat. Cell Biol. 2:76-83.

25. Okada, H., et al. 1998. Novel cis-acting elements in the FSP1 gene regulate fibroblast-specific transcription. Am. J. Physiol. 275:306-314.

26. Gu, H., Zou, Y.R., and Rajewsky, K. 1993. Independent control of immunoglobulin switch recombination at individual switch regions evidenced through Cre-loxP-mediated gene targeting. Cell. 73:1155-1164.

27. Schaffner, D.L., et al. 1993. Targeting of the rasT24 oncogene to the proximal convoluted tubules in transgenic mice results in hyperplasia and polycystic kidneys. Am. J. Pathol. 142:1051-1060.

28. Makino, Y., et al. 2002. Impaired T cell function in RANTES-deficient mice. Clin. Immunol. 102:302-309.

29. Soriano, P. 1999. Generalized LacZ expression with the ROSA26 Cre reporter strain. Nat. Genet. 21:70-71.

30. Moriyama, T., et al. 1998. Up-regulation of HSP47 in the mouse kidneys with unilateral ureteral obstruction. Kidney Int. 54:110-119.

31. Klahr, S., and Morrissey, J. 1998. The role of growth factors, cytokines, and vasoactive compounds in obstructive nephropathy. Semin. Nephrol. 18:622-632.

32. Nagai, N., et al. 2000. Embryonic lethality of molecular chaperone hsp47 knockout mice is associated with defects in collagen biosynthesis. J. Cell Biol. 150:1499-1506.

33. Neilson, E.G., et al. 1984. Spontaneous interstitial nephritis in kdkd mice. I. An experimental model of autoimmune renal disease. J. Immunol. 133:2560-2565.

34. Kelman, Z. 1997. PCNA: structure, functions and interactions. Oncogene. 14:629-640.

35. Slack, J.M., and Tosh, D. 2001. Transdifferentiation and metaplasiaswitching cell types. Curr. Opin. Genet. Dev. 11:581-586.

36. Sun, D., Baur, S., and Hay, E.D. 2000. Epithelial-mesenchymal transformation is the mechanism for fusion of the craniofacial primordia involved in morphogenesis of the chicken lip. Dev. Biol. 228:337-349.

37. Hay, E.D. 1995. An overview of epithelio-mesenchymal transformation. Acta Anat. 154:8-20.

38. Owen, M., and Friedenstein, A.J. 1988. Stromal stem cells: marrowderived osteogenic precursors. Ciba Found. Symp. 136:42-60.

39. Garrett, D.M., and Conrad, G.W. 1979. Fibroblast-like cells from embryonic chick cornea, heart, and skin are antigenically distinct. Dev. Biol. 70:50-70.

40. Schor, S.L., and Schor, A.M. 1987. Clonal heterogeneity in fibroblast phenotype: implications for the control of epithelial-mesenchymal interactions. Bioessays. 7:200-204.

41. Dugina, V., Alexandrova, A., Chaponnier, C., Vasiliev, J., and Gabbiani, G. 1998. Rat fibroblasts cultured from various organs exhibit differences in alpha-smooth muscle actin expression, cytoskeletal pattern, and adhesive structure organization. Exp. Cell Res. 238:481-490.

42. Muller, G.A., and Strutz, F.M. 1995. Renal fibroblast heterogeneity. Kidney Int. Suppl. 50:S33-S36.

43. Alvarez, R.J., et al. 1992. Biosynthetic and proliferative characteristics of tubulointerstitial fibroblasts probed with paracrine cytokines. Kidney Int. 41:14-23.

44. Fan, J.M., et al. 1999. Transforming growth factor-beta regulates tubu- lar epithelial-myofibroblast transdifferentiation in vitro. Kidney Int 56:1455-1467.

45. Desmouliere, A., Geinoz, A., Gabbiani, F., and Gabbiani, G. 1993. Transforming growth factor-beta 1 induces alpha-smooth muscle actin expression in granulation tissue myofibroblasts and in quiescent and growing cultured fibroblasts. J. Cell Biol. 122:103-111.

46. Yang, J., and Liu, Y. 2001. Dissection of key events in tubular epithelial to myofibroblast transition and its implications in renal interstitial fibrosis. Am. J. Pathol. 159:1465-1475.

47. Breen, E., Falco, V.M., Absher, M., and Cutroneo, K.R. 1990. Subpopulations of rat lung fibroblasts with different amounts of type I and type III collagen mRNAs. J. Biol. Chem. 265:6286-6290.

48. Rossert, J., Eberspaecher, H., and de Crombrugghe, B. 1995. Separate cisacting DNA elements of the mouse pro-alpha 1(I) collagen promoter direct expression of reporter genes to different type I collagen-producing cells in transgenic mice. J. Cell Biol. 129:1421-1432.

49. Goldring, S.R., Stephenson, M.L., Downie, E., Krane, S.M., and Korn, J.H. 1990. Heterogeneity in hormone responses and patterns of collagen synthesis in cloned dermal fibroblasts. J. Clin. Invest. 85:798-803.

50. Jelaska, A., Strehlow, D., and Korn, J.H. 1999. Fibroblast heterogeneity in physiological conditions and fibrotic disease. Semin. Immunopathol. 21:385-395.

51. Serini, G., and Gabbiani, G. 1999. Mechanisms of myofibroblast activity and phenotypic modulation. Exp. Cell Res. 250:273-283.

52. Tang, W.W., Van, G.Y., and Qi, M. 1997. Myofibroblast and alpha 1 (III) collagen expression in experimental tubulointerstitial nephritis. Kidney Int. 51:926-931.

53. Eyden, B. 2001. The myofibroblast: an assessment of controversial issues and a definition useful in diagnosis and research. Ultrastruct. Pathol. 25:39-50.

54. Poulsom, R., et al. 2001. Bone marrow contributes to renal parenchymal turnover and regeneration. J. Pathol. 195:229-235.

55. Cornacchia, F., et al. 2001. Glomerulosclerosis is transmitted by bone marrow-derived mesangial cell progenitors. J. Clin. Invest. 108:1649-1656. doi:10.1172/JCI200112916.

56. Barasch, J., et al. 1999. Mesenchymal to epithelial conversion in rat metanephros is induced by LIF. Cell. 99:377-386.

57. Huss, R. 2000. Isolation of primary and immortalized CD34-hematopoietic and mesenchymal stem cells from various sources. Stem Cells. 18:1-9.

58. Bi, L.X., Simmons, D.J., Hawkins, H.K., Cox, R.A., and Mainous, E.G. 2000. Comparative morphology of the marrow sac. Anat. Rec. 260:410-415

59. Simmons, D.J. 1996. The in vivo role of bone marrow fibroblast-like stromal cells. Calcif. Tissue Int. 58:129-132.

60. Weiss, L., and Geduldig, U. 1991. Barrier cells: stromal regulation of hematopoiesis and blood cell release in normal and stressed murine bone marrow. Blood. 78:975-990.

61. Huss, R., Hong, D.S., McSweeney, P.A., Hoy, C.A., and Deeg, H.J. 1995 Differentiation of canine bone marrow cells with hemopoietic characteristics from an adherent stromal cell precursor. Proc. Natl. Acad. Sci. USA. 92:748-752.

62. Huss, R. 2000. Perspectives on the morphology and biology of CD34negative stem cells. J. Hematother. Stem Cell Res. 9:783-793. 